\title{
Epidemiologia do Líquen Escleroso Vulvar em um Hospital Escola do Nordeste do Brasil
}

Epidemiology of the Vulvar Lichen Sclerosus in a Northeast of Brazil School Hospital

Epidemiología del Líquen Escleroso Vulvar en un Hospital Escuela del Nordeste de Brasil

Jose Humberto Belmino Chaves ${ }^{1}$

Alessandra Plácido Lima Leite ${ }^{2}$

Antônio Fernando de Sousa Bezerra ${ }^{3}$

Amanda Vasconcelos de Carvalho Souza ${ }^{4}$

Júlio Cesar Barboza Lima ${ }^{5}$

\section{Resumo}

Objetivo: Avaliar a frequência do Líquen Escleroso Vulvar (LEV) em mulheres atendidas no setor de Ginecologia em um Hospital Universitário. Método: Trata-se de um estudo epidemiológico, transversal e retrospectivo, realizado com 17 pacientes mulheres portadoras de Líquen Escleroso Vulvar, maiores de 18 anos, atendidas no setor de ginecologia de um Hospital Universitário, no período compreendido entre janeiro de 2012 a dezembro de 2016, com laudo anatomopatológico confirmando a patologia. Para a obtenção dos dados foi utilizado um questionário como instrumento de coleta, com seis questões, onde foram avaliados aspectos epidemiológicos como incidência, idade, sintomas, entre outros. Resultados: Observou-se a frequência

\footnotetext{
${ }^{1}$ Médico. Professor da Disciplina de Ginecologia da FAMED /UFAL. Doutor em Comunicação em Saúde pela Universidade Aberta de Lisboa-Portugal e em Bioética e Ética Médica pela Faculdade de Medicina da Universidade do Porto-Portugal. Autor correspondente: Campus A. C. Simões. Av. Lourival Melo Mota, s/n. Tabuleiro dos Martins. 57072-900. Maceió, Al, Brasil. E-mail: jhbchaves@uol.com.br ${ }^{2}$ Médica. Graduada em Medicina pela UNCISAL. Professora da Disciplina de Ginecologia da Faculdade de Medicina FAMED /UFAL. Doutora em Ciências pela UNIFESP.

${ }^{3}$ Médico. Graduado em Medicina pela UFAL. Professor da Disciplina de Patologia da FAMED /UFAL. Doutor em Patologia pelo Hospital Universitário Antônio Pedro, pela Universidade Federal Fluminense.

${ }^{4}$ Graduanda em Medicina pela UFAL.

${ }^{5}$ Graduando em Medicina pela UFAL.
}

Recebido: Abr./2018 - Aceito: Ago./2018. 
de mulheres com Líquen Escleroso Vulvar por anatomopatológico de 17 pacientes. Quanto à raça, a maioria das pacientes se autodeclararam da raça branca $(52,9 \%)$. No quesito faixa etária, as mulheres com idades entre 40 e 60 anos contabilizaram $82 \%$ do universo estudado. $\mathrm{O}$ prurido e o ardor foram os sintomas mais citados, com valores de $64 \%$ e 52,9\%, respectivamente. Com relação à coinfecção, apenas uma $(5,8 \%)$ paciente tinha o diagnóstico do Vírus Papiloma Humano registrado em seu prontuário. No item sobre a evolução para Carcinoma de Células Escamosas da Vulva, duas pacientes $(11,7 \%)$ foram diagnosticadas com essa neoplasia maligna. Conclusão: Foi observado que os dados encontrados neste estudo, em sua maioria, apresentam concordância com outros estudos realizados relativos ao tema. De acordo com dados colhidos em nossa amostra, as mulheres brancas que estão na pré e pós menopausa estão mais propensas a adquirirem Líquen Escleroso Vulvar. A correlação com o Papiloma Vírus Humano não foi observado em nossos resultados, semelhantemente aos consultados na literatura. Identificou-se anatomopatológico de Carcinoma de Células Escamosas da Vulva e 02 casos da população estudada.

\section{Descritores: Líquen Escleroso Vulvar; Vulvar Diseases; Mulheres.}

\section{Abstract}

Objective: To evaluate the frequence of Vulvar Sclerosis Lichen (LEV) in women attended in the Gynecology department of the University Hospital. Method: This is a cross-sectional and retrospective study of 17 female patients, older than 18 years, with the confirmed diagnosis of Lichen Sclerosus Vulvar treated in the gynecology department of the University Hospital from January of 2012 to December of 2016, each patient had an anatomopathological report confirming the pathology. To obtain the data, a questionnaire with six questions was used, and sociodemographic and clinical variables such as incidence, age and symptoms were evaluated. Results: It was verified that the incidence of women with Vulvar Sclerosis Lichen with diagnosis confirmed by anatomopathology was of 17 patients. Regarding the race, the majority of the patients declared themselves as white $(52,9 \%)$. Women between 40 and 60 years accounted for $82 \%$ of our total sample. Pruritus and burning were the most frequently reported symptoms, 
$64 \%$ and $52.9 \%$, respectively. According to the medical records, only one $(5.8 \%)$ patient was coinfected with the Human Papillomavirus. The item about the progression to Vulvar Squamous Cell Carcinoma showed that two patients (11.7\%) were diagnosed with this malignant neoplasm. Conclusion: It was observed that the data found in this study, for the most part, are in accordance with other studies about this subject. White women who are pre and post menopausal are more likely to develop Vulvar Sclerosis. The correlation with the Papilloma Virus seems not to be clarified in the literature, lacking further studies. Vulva's squamous cell carcinoma is the main malignant neoplasm associated with this pathology.

\section{Descriptors: Vulvar Lichen Sclerosus; Doenças da Vulva; Women.}

\section{Resumen}

Objetivo: Evaluar la frecuencia del Líquen Escleroso Vulvar (LEV) en mujeres atendidas en el sector de Ginecología del Hospital Universitario. En el presente trabajo se analizaron los resultados obtenidos en el estudio de la enfermedad de Chagas, en el que se observó un aumento de la mortalidad por cáncer de próstata, de 2012 a diciembre de 2016, con laudo anatomopatológico confirmando la patología. Para la obtención de los datos se utilizó un cuestionario como instrumento de recolección, con seis cuestiones, con las cuales se evaluaron aspectos epidemiológicos como incidencia, edad, sintomas entre otros. Resultados: Se verificó la presencia de mujeres con Líquen Escleroso Vulvar con diagnóstico comprobado por anatomopatológico fue de 17 pacientes. Con respecto a la raza, la mayoría de las pacientes se autodeclararon de la raza blanca (52,9\%). En el caso del grupo de edad, las mujeres con edades entre 40 y 60 años contabilizaron el $82 \%$ del universo estudiado. El prurito y el ardor fueron los síntomas más citados, con valores de 64\% y 52,9\%, respectivamente. Con respecto a la coinfección, sólo una $(5,8 \%)$ paciente tenía el diagnóstico del Virus Papiloma Humano registrado en su prontuario. En el item sobre la evolución para Carcinoma de Células Escamosas de la Vulva, dos pacientes (11,7\%) fueron diagnosticados con esa neoplasia maligna. Conclusión: Se observó que los datos encontrados en este estudio, en su mayoría, presentan concordancia con otros estudios realizados sobre el tema. Las mujeres blancas que están en 
la pre y post menopausia son más propensas a desarrollar Líquen

Escleroso Vulvar. La correlación con el Papiloma Virus parece no estar aclarada en la literatura, carece de más estudios. Y el Carcinoma de Células Escamosas de la Vulva es la principal neoplasia maligna asociada a esa patología.

\section{Descriptores: Liquen Escleroso}

Vulvar; Enfermedades de la

\section{Vulva; Mujeres.}

\section{Introdução}

O Líquen Escleroso Vulvar (LEV) é uma patologia dermatológica crônica e benigna. É caracterizado por um processo de inflamação acentuada com formação de máculas e pápulas poligonais de coloração branconacaradas com espículas córneas que confluem formando placas hipocrômicas, deixando a pele com aspecto apergaminhado ${ }^{(1,2)}$. Sua etiologia é desconhecida, acredita-se que há uma provável associação com desordens autoimunes, genética, hormonal, infeccioso, neste caso relacionado ao Vírus Papiloma Humano (HPV), e por traumas repetidos no local afetado, na patogênese desta doença ${ }^{(3)}$.
Foi descrito inicialmente no século XIX por Hallopeau e Darier, que consideravam a doença como uma variante do líquen crônico ${ }^{(4)}$. São conhecidos como fatores desencadeantes: infecções genitais prévias, alterações hormonais e trauma local. Porém, sabe-se que as lesões podem surgir sem qualquer alteração anterior ou patologia associada, por isso acredita-se no fator autoimune ${ }^{(1)}$. O Líquen Escleroso Vulvar tem sido associado com doenças autoimunes em até $28 \%$ dos pacientes. A tireoidite autoimune é o distúrbio autoimune concomitante mais comum, seguido pela alopecia areata, vitiligo e anemia perniciosa $^{(5)}$.

O Líquen Escleroso Vulvar está mais associado ao sexo feminino, em uma taxa que pode variar de 6 a 10 mulheres para cada homem acometido, brancas e pós-menopausadas entre 40 e 60 anos ${ }^{(6)}$. É uma dermatose crônica, progressiva, esclerosante e inflamatória. Tem predominância ano-genital (83$98 \%$ ), porém pode ter acometimento extragenital ${ }^{(7)}$. Os sintomas descritos são prurido, irritação, dor na relação sexual, disúria, além da presença das placas hipocrômicas na região vulvar, além disso, pode haver formação de fissuras na pele em decorrência de 
relações sexuais. A sensação de prurido e calor local é uma queixa frequente da maioria das pacientes afetadas ${ }^{(8)}$. Esse conjunto de sintomas pode causar uma grande morbidade e redução da qualidade de vida. Diante do quadro clínico compatível com Líquen Escleroso Vulvar, o diagnóstico é confirmado por biópsia da lesão ${ }^{(9)}$.

O exame histopatológico é de extrema importância para a confirmação da doença e para excluir outras patologias vulvares que atuam como diagnóstico diferencial do Líquen Escleroso Vulvar. Esses diagnósticos diferenciais incluem: dermatite de contato, vulvovaginite atrófica, líquen escleroso, líquen plano e neoplasia intraepitelial vulvar. Essas condições são frequentemente diagnosticadas erroneamente pelos profissionais. Portanto, um exame completo e uma observação cuidadosa durante a terapia inicial; além de uma vigilância contínua são necessários para garantir um diagnóstico preciso e um tratamento adequado ${ }^{(10)}$.

A complicação mais preocupante, quando atinge a região vulvar e que possui frequência relevante, é o Carcinoma de Células Escamosas. Essa neoplasia está presente em aproximadamente 3 a $5 \%$ dos casos de
Líquen Escleroso Vulvar e está descrita a relação desta patologia com a infecção pelo Vírus Papiloma Humano (HPV). Esta associação é aceita como um contribuinte essencial para $\mathrm{o}$ desenvolvimento de neoplasias de vulva, mostrando uma forte interação do vírus em células e progressão neoplásica ${ }^{(11) .}$

O Líquen Escleroso Vulvar é uma doença incurável, porém tratável. Uma resposta à terapêutica traduz-se igualmente por uma reversão ou melhoria dos aspectos histológicos. Os objetivos do tratamento incluem o alívio dos sintomas, prevenir as deformações anatômicas e, teoricamente, a transformação maligna. $\mathrm{O}$ interesse do tratamento nas assintomáticas é discutível. Atualmente, o tratamento padrão do Líquen Escleroso Vulvar é feito com o uso de corticoides de alta potência, e, na maioria dos estudos, foram realizados com Propionato de Clobetasol. Os corticoides têm efeito anti-inflamatório, antipruriginoso e vasoconstrictor, não havendo estudos que comprovem vantagem de um corticoide sobre outro, desde que pertençam a mesma classe ${ }^{(12)}$.

O presente estudo se justifica em decorrência do impacto que tal patologia causa na qualidade de vida 
das pacientes afetadas por ela, além da escassez de dados epidemiológicos regionais acerca do tema, objetivandose verificar a frequência e o perfil epidemiológico das pacientes com Líquen Escleroso Vulvar, diagnosticado por biópsia, atendidas no setor de ginecologia de um determinado Hospital Escola do Nordeste brasileiro no período de janeiro de 2012 a dezembro de 2016.

\section{Método}

O presente trabalho trata-se de uma pesquisa epidemiológica, descritiva, transversal. Os dados foram coletados através da análise de prontuários. Foram incluídos no estudo os dados dos prontuários que se enquadravam na pesquisa ou seja, pacientes mulheres portadoras de Líquen Escleroso Vulvar, maiores de 18 anos, atendidas no setor de ginecologia de um Hospital Universitário da região Nordeste, no período compreendido entre janeiro de 2012 a dezembro de 2016, com laudo anatomopatológico confirmando a patologia. Foram excluídas pacientes portadoras de Líquen Escleroso maiores de 18 anos, que não dispunham de um laudo anatomopatológico definitivo desta patologia.

Esta pesquisa foi submetida e aprovada pelo Comitê de Ética em Pesquisa da Universidade Federal de Alagoas e, posteriormente, aprovada pela Plataforma Brasil, sob parecer de número 2.429.427, seguindo os padrões éticos como rege a Resolução 510/16 do Conselho Nacional de Saúde.

A coleta de dados foi realizada no período de janeiro de 2018 a fevereiro de 2018 no setor de patologia do Hospital Escola e por meio do Serviço de Arquivo Médico (SAME). Dos 21 prontuários analisados, dois não cumpriram os critérios de inclusão e outros dois não foram encontrados no Serviço de Arquivo Médico. Assim, a amostra foi constituída pelos dados contidos nos prontuários de 17 pacientes.

Para a obtenção dos dados foi utilizado um questionário como instrumento de coleta, com seis questões, onde foram avaliados aspectos epidemiológicos como idade e raça; assim como os sintomas da patologia em questão, coinfecção com o Vírus Papiloma Humano (HPV), número de gestações por mulher, antecedentes de Líquen Escleroso e evolução para 
Carcinoma de Células Escamosas da Vulva.

\section{Resultados}

As características das pacientes portadoras de Líquen Escleroso Vulvar que envolvem idade e raça e que foram atendidas no setor de ginecologia de hospital universitário são apresentadas na tabela 1. Observa-se a predominância de mulheres com idades entre 40 e 60 anos, contabilizando $82 \%$ do universo estudado. Além disso, constata-se que a maioria, com 52,9\% da amostra, se autodeclarou branca; e $47 \%$ se declarou ser parda. A raça negra não foi evidenciada nos prontuários.

\section{Tabela 1 - Caracterização das Pacientes com Líquen Escleroso Vulvar Atendidas em um Hospital Escola}

\begin{tabular}{lccc}
\hline \multicolumn{2}{c}{ Variável } & $\mathbf{n}$ & $\%$ \\
Idade & $<40$ anos & 1 & 6 \\
& $>40 \mathrm{e}<60$ & 14 & 82 \\
\multirow{2}{*}{ Raça } & 2 & 12 \\
& Brancas & 9 & 52,9 \\
& Pardas & 8 & 47 \\
& Negras & 0 & 0 \\
\hline
\end{tabular}

No que concerne aos principais sintomas descritos na clínica de pacientes com Líquen Escleroso Vulvar e sua relação com o Vírus Papiloma Humano (HPV) a tabela 2 mostra suas distribuições, junto as suas frequências entre as mulheres. Dentre a clínica apresentada, o prurido anogenital foi o sintoma predominante, tendo sido referido por $11(64 \%)$ mulheres.
Outro sintoma bastante referido foi o ardor, com resultado de $52,9 \%$ das pacientes. A dor, a dispareunia e o calor local foram outros sintomas relatados em menor quantidade. Com relação à infecção concomitante com o Vírus Papiloma Humano, apenas uma $(5,8 \%)$ paciente tinha o diagnóstico do Papiloma Vírus registrado em seu prontuário. Nas demais $(94,1 \%)$, não foram encontrados registros da infecção pelo vírus nos prontuários. 


\section{Tabela 2 - Clínica das Pacientes Portadoras de Líquen Escleroso Vulvar e a} Coinfecção com o Vírus Papiloma Humano (HPV)

\begin{tabular}{|c|c|c|c|}
\hline \multicolumn{2}{|c|}{ Variável } & n & $\%$ \\
\hline \multirow[t]{5}{*}{ Clínica } & Prurido & 11 & 64 \\
\hline & Ardor & 9 & 52,9 \\
\hline & Dor & 4 & 23,5 \\
\hline & Dispareunia & 2 & 11 \\
\hline & Calor local & 1 & 5,8 \\
\hline \multirow{2}{*}{$\begin{array}{c}\text { Infecção por } \\
\text { HPV }\end{array}$} & Presente & 1 & 5,8 \\
\hline & Ausente & 16 & 94,1 \\
\hline
\end{tabular}

A tabela 3 exibe a quantidade de mulheres diagnosticadas com Líquen Escleroso Vulvar que evoluíram com Carcinoma de Células Escamosas da Vulva. Dentre os dados coletados, contabilizou-se duas pacientes $(11,7 \%)$ diagnosticadas com esse tipo de neoplasia registrada por meio de anatomopatológico em prontuário médico.

Tabela 3 - Pacientes portadoras de Líquen Escleroso Vulvar que evoluíram posteriormente com Carcinoma de Células Escamosas da Vulva

\begin{tabular}{lcc}
\hline \multicolumn{1}{c}{ Variável } & $\mathbf{n}$ & $\mathbf{\%}$ \\
$\begin{array}{l}\text { Carcinoma de } \\
\text { Células } \\
\begin{array}{c}\text { Escamosas da } \\
\text { Vulva }\end{array}\end{array}$ & 2 & 11,7 \\
\hline
\end{tabular}

As mesmas, se encontravam com idades entre 40 e 60 anos.

Discussão
Verifica-se neste estudo o predomínio de mulheres portadoras de Líquen Escleroso Vulvar que se encontram na faixa etária entre 40 e 60 anos, no período de pré e pós menopausa (tabela 1). Esta patologia 
pode ocorrer em qualquer idade, período de vida, raça ou localização, sendo, contudo, mais comum antes da puberdade, entre a quarta e a sexta década de vida e após a menopausa ${ }^{(13)}$. Foi percebido em alguns estudos que a incidência possui aspecto bimodal, fazendo um pico pré-púbere e um pósmenopausa. Diante desse acometimento em fases hipoestrogênicas, levantam-se questionamentos sobre a atuação hormonal na patogênese da doença. No entanto, a etiologia continua incerta ${ }^{(14)}$.

Evidencia-se na pesquisa que a maioria das pacientes se autodeclararam da raça branca $(52,9 \%)$, o que corrobora com o achado na literatura acerca deste aspecto. A incidência parece ser idêntica em todo o planeta, aparentemente sem preferências raciais. Alguns estudos apontam para que a incidência possa ser ligeiramente superior nos indivíduos de raça branca (12), o que ficou demonstrado no estudo, com uma porcentagem de autodeclaradas pardas de $47 \%$, bem próxima do valor das mulheres que se declararam brancas.

Com relação à clínica, o estudo traz o prurido como sintoma mais relatado entre as pacientes (tabela 2). Outros sintomas, tais como o ardor, a dor e a dispareunia também puderam ser demonstrados na presente pesquisa.
Essas sintomatologias podem acarretar uma qualidade de vida mais restrita a essas pacientes, além de ser, na maioria das vezes, o motivo pelo qual as mulheres costumam recorrer ao atendimento da ginecologia. Pode-se perceber com a clínica descrita pelas pacientes que as manifestações ocorreram, em $100 \%$ dos casos, na região anogenital.

O prurido como o sintoma mais comumente relatado, ocorre como consequência do frequente trauma determinado pelo ato de coçar, podendo aparecer fissuras, rachaduras, sangramentos, ulcerações e infecções locais. Além disso, pode haver dor, irritação e ardor, acompanhados geralmente de lesões vulvares com alterações anatômicas, eritema e descamação.

Muitas pacientes queixam-se também de dispareunia, disúria, sangramento vaginal, anal ou genital, estenose ou fusão labial, carregando assim um grande potencial para atrofia, levando a um prejuízo funcional significativo $(15,16)$. Parte dessas alterações ocorrem pelo fato de que no Líquen Escleroso Vulvar, histologicamente, a epiderme é escassa com hiperqueratose, homogeneização do colágeno entre derme e epiderme; além de infiltrado linfocitário ${ }^{(17)}$. 
Outro fator, muito abordado pela literatura, é a presença da infecção pelo Vírus Papiloma Humano (HPV) como um dos fatores de risco para o Líquen Escleroso Vulvar. Esse quesito também foi pesquisado neste presente estudo, onde dentre os dados contidos nos prontuários das 17 pacientes da amostra, foi encontrado apenas um registro de infecção pelo presente vírus entre as mulheres com o diagnóstico de Líquen Escleroso Vulvar (tabela 2). O que ocorre é que uma das teorias mais antigas defende que haja um agente infeccioso envolvido nesta patologia.

Os principais suspeitos apontados são os bacilos álcool-ácido resistentes, a espiroqueta Borrelia burgdorferi, o Vírus Papiloma Humano e o vírus da Hepatite C. O papel do Papiloma Vírus é igualmente controverso. Em estudos publicados em mulheres menopausadas foi encontrado o vírus de alto risco oncogênico (tipos 16 e 18) apenas numa pequena parte das mulheres com Líquen Escleroso Vulvar ${ }^{(12)}$. Outro estudo nos Estados Unidos realizado com mulheres diagnosticadas com Líquen Escleroso, nenhuma delas apresentavam infecção pelo vírus ${ }^{(18)}$.

Portanto, isso se assemelharia ao achado da presença do Papiloma Vírus em apenas uma paciente da amostra na pesquisa. Porém, é preciso ressaltar que essa associação entre $\mathrm{o}$ vírus $\mathrm{e}$ a patologia ainda gera muita discussão na literatura e ainda não está comprovado cientificamente que o mesmo possa ser de fato fator de risco para parte dos casos de Líquen Escleroso. Assume-se que, em sua maioria, os oncogenes da p53 (proteína citoplasmática), a inflamação crônica e o dano oxidativo ao DNA são responsáveis pela transformação maligna ${ }^{(19)}$.

No quesito evolução para Carcinoma de Células Escamosas da Vulva, dentre as mulheres portadoras de Líquen Escleroso Vulvar da amostra, foram encontrados dois registros $(11,7 \%)$ de anatomopatológicos desta neoplasia no prontuário de duas pacientes (tabela 3 ).

Estudos com grandes grupos de mulheres com Líquen Escleroso mostraram que o risco para Carcinoma de Células Escamosas da Vulva é de cerca de 4 a $5 \%$, e em estudos longitudinais de casos-controle a proporção deste tipo de carcinoma nessas mulheres parece ser 317 vezes maior que em uma mulher da mesma idade que não possui a patologia de Líquen Escleroso Vulvar (20).

O Carcinoma de Células Escamosas é a neoplasia maligna vulvar mais comum, constituindo cerca de $90 \%$ dos casos de mulheres diagnosticadas 
com câncer de vulva. É encontrada, habitualmente, entre a sexta e a sétima década de vida, comumente após a menopausa, e em estágios avançados $(13,21)$. Contrariamente, no nosso estudo, esta complicação foi diagnosticada em uma faixa etária mais precoce, entre 40 e 60 anos de vida.

Em termos da etiopatogenia deste tipo de neoplasia maligna, o papel do Vírus Papiloma Humano no desenvolvimento do Carcinoma de Células Escamosas Vulvar tem sido tema de grandes estudos e debates. Estudos defendem a existência de dois tipos diferentes desta neoplasia, com mecanismos carcinogênicos distintos: um dependente do Papiloma Vírus, especialmente os tipos 16 e 18 , encontrado em mulheres jovens; e outro independente do Papiloma Vírus, associado ao Líquen Escleroso Vulvar (com ou sem hiperplasia escamosa) em mulheres de faixa etária acima da quarta década de vida ${ }^{(13,22)}$.

Este último corrobora com único achado da neoplasia maligna no presente estudo, pois a paciente em questão não tinha evidência histológica de infecção pelo Papiloma Vírus em seu prontuário, além de se encontrar na faixa etária acima dos 40 anos. Portanto, esta é uma neoplasia que tem uma grande importância de ser diagnosticada o mais precocemente possível, pois quando não tratada de forma rápida, a progressão para o Carcinoma de Células Escamosas invasivo ocorre, geralmente, dentro de seis meses ${ }^{(23)}$.

\section{Conclusão}

Os resultados anatomopatológicos encontrados nessa pesquisa, apresentam concordância com outros estudos realizados na literatura. A amostra composta por mulheres na sua maioria brancas, no período de pré e pós menopausa, prurido e ardor são os sintomas mais referidos e a dermatose em questão não apresentou associação com o Vírus Papiloma Humano.

Apesar do Líquen Escleroso Vulvar, ainda ser uma patologia de diagnóstico tardio, mesmo sendo uma doença de evolução crônica, a identificação da progressão de dois casos para câncer de vulva, reforça essa doença como sendo a principal via carcinogenética para o câncer de vulva. Portanto, um diagnóstico precoce e um acompanhamento sistemático são de extrema relevância para que se conheça a história natural dessa doença, proporcionando meios para a prevenção da sua evolução para o câncer genital. 


\section{Referências}

1. Peixoto AB, Vilela GM, Luz AT, Silva LG. Líquen escleroso e atrófico em criança com história de abuso sexual: Relato de Caso. Rev. Bras. Clin. Med. 2013; 11 (1): 92-3.

2. Cooper SM, Arnold SJ. Vulvar lichen sclerosus. UpToDate. 2016.

3. Della PG, Bozzini N, Cabrera H. Síndromes asociados a alteración del colágeno tipo IV: síndrome de Alport y liquen escleroso y atrófico. Dermatol. Pediatr. Lat. 2014; 2 (2): 139-43.

4. Nóbrega MM, Cabral F, Corrêa MC, Barcaui CB, Bressan AL, Gripp AC. Líquen escleroso associado à esclerodermia localizada: contribuição da dermatoscopia. A. Bras. Dermatol. 2016; 91 (4): 534-6.

5. Fruchter R, Melnick L, Pomeranz, MK. Lichenoid vulvar disease: A review. International Journal of Women's Dermatology. 2017; 3 (1): 58-64.

6. Tavares E, Martins C, Teixeira J. Dermatoses vulvares inflamatórias. Revista da SPDV 2011; 69 (4): 561-71.

7. Guerra A. Liquen escleroso. Actas. Dermosifiliogr. 2013; 10 (94): 633-41.

8. Carmona FLP, Díaz EC, Cano MDCG, Arrabal MAP. Estudio epidemiológico de los pacientes com liquen escleroatrófico genital. Resultados preliminares. Rer. Int. Androl. 2014; 12 (2): 80-3.

9. Pascual MG, Martín, FJV, Estebaranz JL. Lichen Sclerosus and Squamous Cell Carcinoma. Actas. Dermosifiliogr. 2012; 1 (103): 21-8.

10. Potter JE, Moore, KA. Lichen Sclerosus in a Breast Cancer Survivor on an Aromatase Inhibitor: A Case Report. 2016; 28(4): 592-5.
11. Silva JT, Saddi VA, Ribeiro AA. Marcadores moleculares em carcinomas de vulva associados ao HPV - análise de p16 e ki-67: revisão bibliográfica. Actas. Estudos. 2014; 41 (4): $675-88$.

12. Baptista PV, Duarte S, Freitas D, Costa AR, Beires J. Líquen Escleroso: revisão da literatura. Acta. Obstét. Ginecol. Port. 2007; 1(1): 30-41.

13. Tavares E, Parente J, Gonçalves JCA, Teixeira J, Martins J, Aranha J. Líquen escleroso da vulva - Revisão de 208 casos. Revista da SPDV 2012; 70 (1): 81-9.

14. Nair PA. Dermatosis associated with menopause. J. MidLife Health. 2014; 5(4): 168-75.

15. Kirtschig G, Becker K, Gunthert A, Jasaitiene D, Cooper S, Chi CC, et al. Evidence-based (S3) Guideline on (anogenital) Lichen sclerosus. J Eur Acad Dermatol Venereol. 2015; 29 (10): 1-43.

16. Haefner HK, Aldrich NZ, Dalton VK, Gagné HM, Marcus SB, Patel DA, et al. The impacto of vulvar lichen sclerosus on sexual dysfunction. J Womens Health (Larchmt). 2014; 23 (9): 765-70.

17. Curi1 DG, Roa CL, Aguiar LM, et al. Vulvar lichen sclerosus: description of 5 cases successfully treated with the 2,940nm Erbium-YAG laser. Surg Cosmet Dermatol 2017; 9 (3): 264.

18. Miranda JA, Val ICC. Os três líquens: escleroso, plano e plano erosivo. Artigo de revisão. Estudo realizado na Faculdade de Medicina da Universidade Federal Fluminense (UFF). FEMINA 2014; 42 (2): 66-2. 
19. Kirtschig G. Lichen SclerosusPresentation, Diagnosis and Management. Department of General Medicine and Interprofessional Care, University Hospital Tübingen. Review Article. 2016; 113 (19): 337-43.

20. Sanhueza PR, Yaksic NB, Chahuan KI. Valor de la biopsia vulvar en el diagnostico de liquen escleroso en pacientes con alteraciones vulvares crónicas. Rev. Chil. Obstet. Ginecol. 2004; 69 (3): 199-02.

21. Matthews N, Wong V, Brooks J, Kroumpouzos G. Genital diseases in the mature woman. Clin. Dermatol. 2018; 36 (2): 208-21.

22. Hinten F, Molijn A, Eckhardt L, Massuger LFAG, Quint W, Bult P, et al. Câncer de vulva: duas vias com localização e prognóstico diferentes. Gynecol. Oncol. 2018; 147 (2): 31017.

23. Brodrick B, Belkin ZR, Goldstein AT. Influence of treatments on prognosis for vulvar lichen sclerosus: Facts and controversies. Clin. Dermatol. 2013; 31 (6): 780-6. 